LIAMES 14 - pp. 121-147. Primavera 2014

\author{
Verónica Nercesian \\ (CONICET-Universidad Nacional de Formosa, \\ Argentina)
}

\title{
Procesos fonológicos en el dominio de la palabra wichí (mataguaya)
}

\begin{abstract}
This paper aims to analyze the phonological processes which take place within the word in Wichi (Mataguayan). Derivation, inflection and composition imply the combination of morphemes and, thus, the formation of a different sequence of sounds. This triggers phonological adjustments to satisfy constraints or conditionings of different types: (i) articulatory, (ii) phonotactic (of syllabic structure), (iii) internal sandhi, (iv) semantic transparency and (v) rhythmic. The most frequent phonological processes within the word in Wichi can be classified with respect to these conditionings. As regards (i), palatalization; concerning (ii), vocalic and consonantal epenthesis; as to (iii), obstruent aspiration, sonorant devoicing and hiding of the voiceless glottal fricative. Semantic or morphotactic transparency, (iv), triggers the fortition of consonants on morphological edges. Lastly, as regards rhythmic-discursive conditioning, (v), syllabic reduction or syncope. The phonological word is the domain where phonological processes occur, but at the same time, they contribute to the formation of the word as a phonological and morphological unit satisfying the wordhood requirements of the language.
\end{abstract}

KEYWORDS: Mataguayan; Wichi; Phonology; Wordhood; Phonological processes.

RESUMEN: El propósito de este trabajo es presentar el análisis de distintos procesos fonológicos que ocurren dentro de la palabra en wichí (mataguaya). La derivación, la flexión y la composición implican la combinación de morfemas y, por lo tanto, la formación de una secuencia de sonidos diferente, lo que motiva reajustes fonológicos para satisfacer restricciones o condicionamientos de distintos tipos: (i) articulatorio, (ii) fonotáctico (de estructura silábica), (iii) sandhi interno, (iv) transparencia semántica y (v) rítmico. Los procesos fonológicos más frecuentes dentro de la palabra en wichí pueden agruparse según esos condicionamientos. En relación con (i), la palatalización; en relación con (ii), la epéntesis vocálica y consonántica; con (iii) la aspiración de obstruyentes, el ensordecimiento de sonorantes y el ocultamiento de la glotal fricativa sorda. La transparencia semántica o morfotáctica, (iv), motiva el fortalecimiento de consonantes en límites morfológicos. Por último, en relación con el condicionamiento rítmico-discursivo, (v), la reducción silábica o síncopa. La palabra fonológica es el domino en el que tienen lugar los procesos fonológicos, pero al mismo tiempo estos contribuyen a la formación de la palabra como unidad fonológica y morfológica cumpliendo los requisitos de la integridad léxica de la lengua.

PALABRAS CLAVES: Mataguaya; Wichí; Fonología; Formación de palabras; Procesos fonológicos. 
NERCESIAN - PROCESOS FONOLÓGICOS EN EL DOMINIO DE LA PALABRA WICHÍ...

\section{INTRODUCCIÓN}

Los procesos morfológicos de flexión y formación de palabras pueden acarrear procesos fonológicos de reajustes motivados por condicionamientos de distinta índole, a saber, articulatorios, de estructura de sílaba, de estructura de palabra fonológica, entre otros. Su estudio resulta de gran interés para el conocimiento de la 'palabra' como unidad, la fonología y la interacción entre esta y la morfología. El análisis de dichos procesos en el interior de la palabra en wichí y los factores que los motivan es el propósito principal de este trabajo. Si bien el wichí es una lengua aglutinante y el grado de fusión de morfemas no es alto, existen algunos procesos fonológicos considerablemente regulares y frecuentes que merecen un estudio cuidadoso. Asimismo, a partir de datos de la lengua wichí, se establece una clasificación de los procesos en función de los condicionamientos que los motivan a fin de profundizar la reflexión de la multidimensionalidad de la palabra.

El wichí pertenece a la familia lingüística mataguaya junto con el chorote, el maká y el nivakle, y se habla dentro de la región chaqueña en una vasta extensión geográfica que abarca las provincias de Formosa, Chaco y Salta en Argentina y la ribera del río Pilcomayo en el Departamento de Tarija en Bolivia. En toda la extensión de habla de la lengua wichí se distinguen dos grandes variedades, la bermejeña y la pilcomayeña que deben sus nombres a los ríos Bermejo y Pilcomayo que históricamente habita este pueblo. En el estudio de los procesos fonológicos esta variación es relevante en tanto he registrado algunas diferencias. No obstante, el presente trabajo no se focaliza en esa variación sino en el corpus de datos que pertenece a la variedad del Bermejo conformado -recogidos personalmente en las provincias de Chaco y Formosa-. El corpus se confeccionó, por un lado, con palabras obtenidas mediante la elicitación de listas, y por el otro, a partir del análisis de textos de distintos géneros (narrativos, expositivo-explicativos, legislativo, entre otros).

En la sección $\$ 2$ se presentan algunas generalidades de la fonología wichí para facilitar la lectura de los fenómenos que se analizan en las secciones siguientes. En $\$ 3$ se presentan los procesos fonológicos, los condicionamientos que los motivan y su clasificación. La sección $\$ 3.1$ está centrada en el análisis de la palatalización; §3.2, en la epéntesis vocálica y consonántica; $\$ 3.3$, en la aspiración; $\$ 3.4$, en el ensordecimiento de sonorantes; $\$ 3.5$, en el ocultamiento de la glotal fricativa; $\$ 3.6$, en el fortalecimiento de consonantes y, finalmente, $\$ 3.7$, en la reducción silábica. En la sección $\$ 4$ se muestra en qué medida la palabra constituye un dominio para los procesos a la vez que éstos contribuyen a la formación de la palabra como unidad. Por último, en $\$ 5$ se presentan las conclusiones.

\section{GENERALIDADES DE LA FONOLOGÍA WICHÍ}

El sistema fonológico wichí se caracteriza por su complejo inventario de consonantes que presenta un contraste de tres series según el mecanismo de fonación: plena, aspirada y glotalizada (o eyectiva) y un total de 34 fonemas consonánticos: $/ \mathrm{p} / ; / \mathrm{p}^{\mathrm{p}} / ; / \mathrm{p}^{\mathrm{h}} / ; / \mathrm{t} / ; / \mathrm{t}^{\mathrm{t}} / ;$;

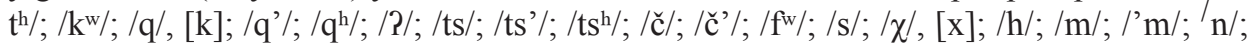
/'n/; /ñ /; /l/; /'’l/; /l/; /j/; /'j/; /j/; /w/;/'w/; /wo/ (Nercesian 2011a). El inventario carece 
del contraste sordo/sonoro en las consonantes oclusivas y fricativas, sólo posee la serie sorda. Entre las consonantes dorsales, el wichí posee dos fonemas uvulares uno oclusivo y uno fricativo (cada uno de ellos presenta una realización velar que ocurre en contextos $\mathrm{V}$ [anterior] _\#, V[anterior] _ C) y un fonema velar labializado. Asimismo, posee dos fonemas glotálicos, oclusivo y fricativo. Otro aspecto interesante del sistema es que distingue entre lateral sonora plena y glotalizada, por un lado, y lateral sonora y lateral fricativa sorda, por el otro. Por último, las semiconsonantes $/ \mathrm{j} / \mathrm{y} / \mathrm{w} /$ se comportan en wichí como consonantes: (i) pueden ser afectadas por el proceso de ensordecimiento provocado por la presencia de la $/ \mathrm{h} /$, al igual que las sonorantes $/ 1 /, / \mathrm{m} /, / \mathrm{n} /$ en límites morfológicos; (ii) ocupan la posición de ataque y coda en la sílaba, y (iii) sirven de consonante epentética en la posición de ataque para interrumpir secuencias de dos vocales contiguas (no admitidas en esta lengua).

En cuanto al inventario vocálico, este está conformado por un total de cinco fonemas orales: /i/;/e/;/a/;/o/; /u/. Las variantes nasalizada y la laringealizada no son fonológicas, dependen del contexto fónico en el que ocurren. Tanto las consonantes nasales como la glotal fricativa sorda /h/ provocan la nasalización de la vocal siguiente. La laringealización se observa cuando la vocal está precedida de la glotal oclusiva sorda o de una consonante eyectiva (Nercesian 2011a).

Desde una perspectiva tipológica, según los parámetros propuestos por el World Atlas of Linguistic Structure (WALS) el inventario consonántico wichí es moderadamente extenso; característica que comparte con un grupo de 116 lenguas del mundo (de un total de 563 lenguas, 90 presentan un inventario pequeño, 121 moderadamente pequeño, 182 un número promedio y 54 uno extenso; cf. Maddieson 2011a). Mientras que el inventario de vocales wichí es un sistema fonémico promedio (cf. Maddieson 2011b).

Con respecto a la estructura silábica, el wichí no manifiesta rasgos sobresalientes. Al contrario, está dentro del parámetro más extendido según las medidas del WALS, presentando una estructura silábica moderadamente compleja (cf. Maddieson 2011c). Las sílabas posibles en wichí son CV, CVC, CCV. En el ataque puede ocurrir cualquier consonante del inventario, plena, glotalizada (o eyectiva) y aspirada. En esta lengua, las sílabas sin ataque no son permitidas. Los ataques complejos son muy poco frecuentes y sólo pueden ser: /tl/, /pl/ y / kl/. En la posición de núcleo silábico pueden ocurrir todas las vocales y las dos sonorantes alveolares $/ \mathrm{n} / \mathrm{y} / 1 /$. No hay núcleos complejos o diptongos. Las secuencias como /aj/, /ej/, /oj/, /uj/ son extremadamente comunes en wichí y ocurren en el interior y en el final de palabra, pero son consideradas como secuencias VC dado que las semiconsonantes son consonantes. Por último, en la posición de coda silábica pueden ocurrir únicamente las consonantes plenas y la labializada, así como también la semiconsonante palatal. No pueden ocurrir en coda segmentos complejos (aspirados, glotalizados o eyectivos), tampoco grupos consonánticos (Nercesian 2011b).

Finalmente, la fonotaxis del wichí permite una gran variedad de posibilidades de secuenciación de consonantes, mientras que las restricciones en la combinatoria de sonidos, en cambio, son pocas. En interior de palabra, como máximo puede haber dos consonantes contiguas en los contornos silábicos: 


$\begin{array}{ll}\text { CV.CV }(C) & * \operatorname{CVC} . C C V(C) \\ \text { CVC.CV }(C) & * C V C C . C V(C) \\ \text { CV.CCV }(C) & * \operatorname{CV} . V(C)\end{array}$

Las fricativas uvular y glotal, $[\chi]$ y $[\mathrm{h}]$, no aparecen en posición de ataque si están precedidas de una consonante: ${ }^{*}[\mathrm{VC} . \chi \mathrm{V}],{ }^{*}[\mathrm{VC} . \mathrm{hV}]$. Las series glotalizadas (y eyectivas) $\mathrm{y}$ aspiradas ocurren generalmente en posición intervocálica. Son muy pocos los casos en que están precedidas por una consonante.

\section{PROCESOS FONOLÓGICOS}

Los procesos de formación de palabras gramaticales que se realizan mediante procesos de morfología concatenativa implican la formación de una palabra fonológica con una estructura secuencial de sonidos diferente. Esta nueva combinación de sonidos puede no satisfacer los condicionamientos mínimos para la buena formación de la palabra fonológica (PalFon) asociada a la palabra gramatical (PalGram). Como consecuencia pueden producirse algunos reajustes. Lingüistas como Booij (1999: 47), Dixon y Aikhenvald (2002: 10ss.), entre otros, sostienen que la PalFon es la contraparte de la PalGram, sin que ello signifique que sean isomórficas. La PalFon es la unidad prosódica que representa la interacción entre los componentes fonológicos y morfológicos de la gramática (Booij y Rubach 1987; Nespor y Vogel 1986; Booij y Lieber 1993, entre otros). Aún más, en cualquier lengua la palabra fonológica se correlaciona sistemáticamente con límites morfológicos (Hall 1999: 15), porque representa la interacción entre los componentes fonológicos y morfológicos, puede contener límites morfológicos internos y ser la contraparte de la PalGram que constituye el dominio para diferentes generalizaciones fonológicas.

La PalFon en wichí es el dominio de ocurrencia de distintos procesos fonológicos de reajuste (cf. Sección $\$ 4$ ). La aspiración de obstruyentes, el ensordecimiento de sonorantes, el ocultamiento de la glotal fricativa, la palatalización, la epéntesis vocálica y consonántica, el fortalecimiento de consonantes y la reducción silábica son los más frecuentes. Si bien estos procesos ocurren en favor de la buena formación de la palabra, los factores que los motivan son de distinta índole. Véase cuadro 1.

Cuadro 1. Procesos fonológicos y condicionamientos

\begin{tabular}{|l|l|}
\hline \multicolumn{1}{|c|}{ Proceso fonológico } & \multicolumn{1}{c|}{ Tipo de condicionamiento } \\
\hline (i) palatalización & articulatorio \\
\hline (ii) epéntesis vocálica y consonántica & fonotáctico (estructura de la sílaba) \\
\hline $\begin{array}{l}\text { (iii) aspiración de obstruyentes } \\
\text { (iv) ensordecimiento de sonorantes } \\
\text { (v) ocultamiento de la glotal fricativa }\end{array}$ & $\begin{array}{l}\text { sandhi interno (combinatoria de sonidos entre } \\
\text { sílabas en límites morfológicos) }\end{array}$ \\
\hline (vi) fortalecimiento de consonantes & transparencia semántica \\
\hline (vii) reducción silábica & rítmico \\
\hline
\end{tabular}


Como se refleja en el cuadro 1, el proceso fonológico (i) es de corte articulatorio, (ii) responde a condicionamientos fonotácticos de estructura silábica, (iii)-(v) están motivados por restricciones de sandhi interno, (vi) ocurre en favor de la transparencia semántica en límites morfológicos dentro de la palabra y, por último, (vii), la reducción silábica, parece estar motivada por factores rítmicos de la frase.

Los tipos de condicionamiento de los procesos fonológicos pueden ser ordenados en función del nivel que hacen prevalecer. El condicionamiento articulatorio que motiva la palatalización (i) da prioridad a la naturaleza articulatoria de los sonidos haciendo prevalecer el nivel del segmento. El condicionamiento fonotáctico de estructura silábica (ii) da prioridad al nivel de la sílaba, mientras que el sandhi interno (iii)-(v) prioriza la combinación de sonidos entre sílabas dentro de la palabra fonológica al añadirse morfemas a la base. La transparencia semántica (vi) prioriza la estructura de la palabra gramatical asociada a la palabra fonológica y provoca la interacción fonología-morfología. Por último, los condicionamientos rítmicos (vii) exceden el nivel del segmento, la sílaba y la palabra fonológica y dan prioridad a la estructura prosódica o métrica de la frase. Esta distribución no implica un ordenamiento de aplicación, sino una clasificación de los procesos según el nivel lingüístico de los factores condicionantes. Ello refleja la multidimensionalidad de la palabra y la interacción de distintos tipos de información lingüística en los procesos morfológicos.

Los procesos mencionados ocurren para y en la formación de palabras, no sólo porque se aplican para asegurar su buena formación, sino también porque ocurren simultáneamente al proceso morfológico de derivación, composición (e incorporación), flexión y por ello también sirven para reconocerla (Nercesian 2011a; en prensa). Incluso, ciertos procesos fonológicos que tienen lugar en el interior de la palabra reconocen información morfológica y pueden ser condicionados por ella; p.ej. el fortalecimiento de consonantes o el bloqueo de los procesos fonológicos. En las secciones siguientes se examinan con detalle cada uno de los procesos mencionados en el cuadro 1.

\subsection{Palatalización}

La palatalización consiste en un proceso de asimilación del punto de articulación de segmentos anteriores y altos que afecta a la consonante contigua. Desde la perspectiva de la fonología de gestos (Browman y Goldstein 1986; 1989; 1992; Saltzman 1995; Saltzman y Kelso 1987) ello se explica por la superposición del gesto oral cuerpo de la lengua especificado como anterior alto por la vocal precedente que se mantiene activo al tiempo que se activa el gesto glotal crítico (en el caso de las consonantes fricativas) o abierto (en el caso de las oclusivas sordas). En wichí, dicho proceso de asimilación se produce en el dominio de la sílaba de derecha a izquierda. Las consonantes velares (oclusiva y fricativa) en posición intervocálica precedidas por /i/ y /e/ adelantan su punto de articulación del velo al paladar duro, (1).

$$
\begin{aligned}
& [\mathrm{k}] \rightarrow[\mathrm{c}] / \text { V[anterior }] \_\mathrm{V} \\
& \text { / V [anterior] } \_\mathrm{hV} \\
& [\mathrm{x}] \rightarrow[\mathrm{S}] / \mathrm{V} \text { [anterior }] \_\mathrm{V} \\
& \text { / V[anterior] _ } \mathrm{hV}
\end{aligned}
$$


Las consonantes uvulares tienen un alófono velar que ocurre precedido de las dos vocales anteriores en posición de coda silábica, las cuales causan el adelantamiento del punto al velo del paladar. En el caso de la palatalización de la fricativa el resultado de la asimilación es un segmento que no forma parte del inventario consonántico de la lengua, la [S]; por el contrario, la palatalización de la oclusiva velar da / ̌̌/. La consonante en coda palatalizada pasa a ocupar la posición de ataque de la sílaba siguiente, como se ilustra en (2).
a. CV.CV.CV+CV.CVC+V CV.CV.CV.CV.CV
[?i.wu.je]+[te.nek] $]+[-\mathrm{a}]$
3SUJ:hacer+canción $+\mathrm{C}$
$\rightarrow \quad$ [?i.wu.te.ne.ča] 'Adora'
b. $\mathrm{CVC}+\mathrm{CV} . \mathrm{CV}$
CV.CV.CV
[jık]+[-hi.la]
$\rightarrow \quad$ [jı.čhi.la]
'Se va a ir'
3SUJ:irse+FUT
c. $\mathrm{CVC}+\mathrm{CVC}$
[jik $]+[=$ hen $]$
3SUJ:irse +PL
$\rightarrow \quad$ [jı.čch
'Se van'
d. $\mathrm{CV} \cdot \mathrm{CVC}+\mathrm{CV}$
CV.CV.CVC
[?i.lex $]+[-e x]$
[?i.le.fex]
'Lava con algo'
3SUJ:lavar+INS

$\rightarrow \quad$ [?i.le.fex]
$\begin{array}{ll} & \text { CV.CV.CV } \\ \rightarrow \quad & \text { ['no.ji.fa] }\end{array}$
e. $\mathrm{CV} \cdot \mathrm{CVC}+\mathrm{CV}$
'este camino'
camino+DEM
f. CV.CVC $+\mathrm{CV}$
CV.CV.CV
[?i.lex $]+[-h u]$
[?i.le.ju]
'Lo limpia por dentro'
3SUJ:lavar+LOC

Nótese que en los ejemplos (2b y c) la glotal fricativa en posición de ataque de los sufijos añadidos es transparente para la asimilación. Mientras que el gesto glotal abierto se activa y desactiva, la especificación de constricción local del gesto cuerpo de la lengua cambia de media alta en la realización de la vocal anterior, al punto palatal (por asimilación de la velar) para pasar al posterior alto. En estos casos, además de la asimilación del punto de articulación, la consonante palatalizada [č] se aspira por la superposición parcial del gesto glotal abierto de las dos consonantes. En el ejemplo (2 e), se produce el ocultamiento de la nasal (fenómeno que ocurre cuando la consonante está precedida por una fricativa) y, como consecuencia, la velar fricativa queda en posición intervocálica y se palataliza. Dado que el proceso de asimilación ocurre en el dominio de la sílaba, la uvular en posición de ataque no adelanta el punto de articulación (ni al velar y ni al palatal) a pesar de estar precedida por una vocal anterior alta (véanse ejemplos en (3).

a. CV.CVC

[ji.qon]

'Le gusta' 
b. CV.CVC

[jr.quj] 'Juega'

c. CV.CV.CV

[?i.qa.na] 'Está acá'

La palatalización también se produce cuando por causa del ocultamiento de la glotal fricativa la velar queda en posición intervocálica, como se ilustra en (4).

(4)

a. $\mathrm{C}+\mathrm{CVC}+\mathrm{CVC}$

$[\mathrm{n}-]+[$ lex $]+[=$ hen $] \quad \rightarrow$

1SUJ+lavar+PL

b. $\mathrm{CV}+\mathrm{CV}+\mathrm{CV}+\mathrm{CV} \cdot \mathrm{CVC}+\mathrm{CV}$

$[$ ha- $]+[$ ?a- $]+[$ qa- $]+[$ tu.wex $]+[-$ hi $] \rightarrow$

$\mathrm{NEG}+2 \mathrm{POS}+\mathrm{CL}+$ tinaja $+\mathrm{NEG}$

c. $\mathrm{CV} \cdot \mathrm{CVC}+\mathrm{CV}+\mathrm{CV}$

[?i.that $]+[-e x]+[-$ hu $] \rightarrow$

3SUJ:mandar+INS+LOC
C..CV.CVC

[n.le.fen]

CV.CV.CV.CV.CV.CV

[ha.Pa.qa.tu.we.fi]

CV.CV.CV.CV

[?i.tha.te.fu]
'No es tu tinaja'

'Yo los lavo'

'Tira algo a alguien'

El proceso morfofonológico de palatalización es una diferencia dialectal en el wichí. Los propios hablantes brindaron ejemplos de contraste con la variedad pilcomayeña de Salta, en los que el proceso de palatalización no ocurre a pesar de encontrarse la velar en los mismos contextos fonológicos que se ha ilustrado antes, p. ej. [jik]+[hen] $\rightarrow$ [jičhen] vs. [jik ${ }^{\mathrm{h} e n]}$. Asimismo, algunos ejemplos presentados en Cleasson (1994) como yikeh 'Él/ella va por algo' (op.cit: 17); nekhiilah 'Él/ella va a venir' (op.cit: 24) no manifiestan palatalización, mientras que en el wichí bermejeño estos son contextos propicios para la ocurrencia de dicho proceso morfofonológico. En otras lenguas mataguayas, el chorote (Gerzenstein 1978), el maká (Gerzenstein 1994) y el nivaclé (Stell 1987), no se ha registrado la palatalización como proceso morfofonológico sincrónicamente activo, mientras que en lenguas guaycurúes como el abipón (Najlis 1966), el mocoví (Gualdieri 1998), el pilagá (Vidal 2001) y el qom o toba (Messineo 2003) procesos de palatalización son altamente recurrentes, con excepción del kaduvéo (Sândalo 1995). También se ha registrado en otras lenguas chaqueñas como el tapiete de la familia tupi-guaraní (González 2005: 80), por lo que podría especularse que sea un rasgo extendido en el área. La recurrencia de este proceso de asimilación en las lenguas guaycurúes y la ausencia del mismo en las mataguayas con excepción de la variedad wichí bermejeña podría ser un indicio de que este último haya incorporado la palatalización por contacto lingüístico con las guaycurúes.

\subsection{Epéntesis vocálica y consonántica}

Los condicionamientos de la estructura de sílaba en wichí pueden motivar procesos de epéntesis. Los segmentos epentéticos se añaden en la silabificación de la palabra cuyos sufijos son menores a una sílaba y pueden ser vocálicos o consonánticos dependiendo del contexto. 
La epéntesis vocálica consiste en añadir una vocal anterior entre la raíz y el sufijo de plural nominal que consiste en una consonante. Cuando la raíz o base termina en vocal, el sufijo ocupa la posición de coda de la última sílaba de la palabra, obsérvense ejemplos en (5). Cuando la raíz termina en consonante, esa posibilidad está restringida dado que el wichí no admite codas complejas, lo que motiva la epéntesis vocálica creando una sílaba nueva, como se ilustra en (6).

\begin{tabular}{|c|c|c|c|c|c|}
\hline a. /-s/ & $\begin{array}{l}{\left[\mathrm{f}^{\text {winču-s}}\right]} \\
\text { [mamse-s] }\end{array}$ & $\begin{array}{l}\text { 'escamas' } \\
\text { 'varones' }\end{array}$ & a. $[-\mathrm{Vs}]$ & $\begin{array}{l}\text { [tołet-e-s] } \\
\text { [tołej-i-s] }\end{array}$ & $\begin{array}{l}\text { 'leñas' } \\
\text { 'nombres' }\end{array}$ \\
\hline b. /-1/ & $\begin{array}{l}\text { [tots'e-1] } \\
\text { [leč'e-1] }\end{array}$ & $\begin{array}{l}\text { 'panzas' } \\
\text { ‘huevos' }\end{array}$ & b. $[-\mathrm{VH}]$ & $\begin{array}{l}{[\text { čos-e-1] }} \\
{[\text { qatets-e-1] }}\end{array}$ & $\begin{array}{l}\text { 'colas' } \\
\text { 'estrellas' }\end{array}$ \\
\hline c. $/-\mathrm{j} /$ & $\begin{array}{l}\text { [ha'lo-j] } \\
\text { [toč'ute-j] }\end{array}$ & $\begin{array}{l}\text { ‘árboles' } \\
\text { ‘orejas' }\end{array}$ & c. $[-\mathrm{Vj}]$ & $\begin{array}{l}{\left[\text { totk }^{w} \mathrm{ej-a-j]}\right.} \\
\text { [tones-e-j] }\end{array}$ & $\begin{array}{l}\text { 'brazos' } \\
\text { 'narices' }\end{array}$ \\
\hline
\end{tabular}

La tendencia es que la vocal epentética asuma los rasgos del segmento vocálico más próximo hacia la izquierda. Nótese que, con excepción de [totk ${ }^{\mathrm{w} e j a j]}$ 'brazos de alguien', la vocal epentética es una anterior alta /i/ si el último segmento de la raíz es la/j/, y es una anterior media /e/, si la última vocal de la raíz es una media: /e/ u /o/.

La epéntesis consonántica consiste en la adición de la semiconsonante palatal /j/ en posición de ataque silábico entre dos vocales contiguas (heterorgánicas u homorgánicas). Este proceso previene las secuencias VV y la ocurrencia de sílabas V no admitidas en la fonotaxis y la estructura silábica del wichí. Al interrumpir la secuencia vocálica mediante la semiconsonante se crean dos sílabas CV: $\mathrm{CVV}(\mathrm{C}) \rightarrow \mathrm{CV} \cdot \mathrm{GV}(\mathrm{C})^{1}$, como se ilustra en (7). La epéntesis ocurre cuando la base termina en vocal y se añade un sufijo que inicia en vocal.

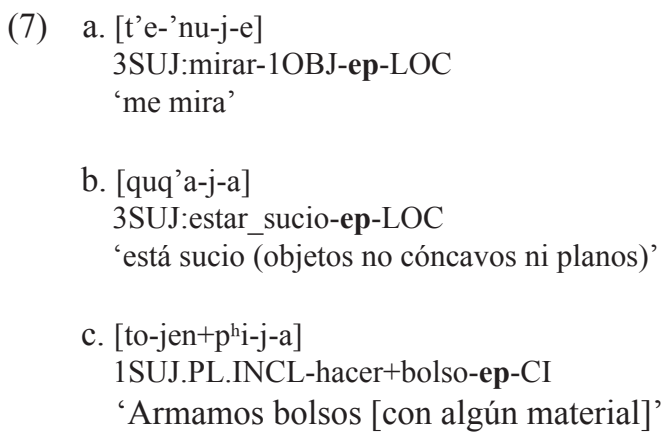

Por último, un segundo proceso de epéntesis consonántica consiste en la realización de un segmento nasal en posición de coda seguida de una consonante y sílaba acentuada. A diferencia de los dos procesos de epéntesis anteriores que responden a restricciones fonotácticas y de estructura de sílaba, este es fonético. Como se observa en los ejemplos

\footnotetext{
${ }^{1}$ La G (glide) se utilizó para consignar las semiconsonates.
} 
presentados en (8), la nasalización de la vocal por efecto de la glotal fricativa o de un segmento aspirado se realiza como segmento nasal con el punto de articulación de la consonante siguiente:
a. [?i-jahĩn-phõ-pex] $\rightarrow$
[Pi.ja.hĩn.ppõm.pex]
'mira hacia arriba varias veces'
b. [nek-phõ-pex]
[ne.pnõm.pex]
'se levanta varias veces'
c. [?ihĩ-lo?]
[?i.hĩn.lo?]
'está enfrente'
d. [jinũ-lo?]
[ji.hũn.lo?]
'está viniendo'

En la primera columna se separaron con guiones los morfemas, en la segunda columna se resaltó en negrita la nasal epentética en coda y se separaron las sílabas mediante puntos. En los ejemplos (8 a y b) la nasalización se realizó como [m], puesto que la consonante que le sigue es bilabial, mientras que en los ejemplos ( 8 c y d) se realizó como [n], dado que la consonante contigua es alveolar.

\subsection{Aspiración}

La aspiración de las consonantes en wichí puede producir dos tipos de segmentos según el contexto fónico: consonantes con fonación aspirada, p.ej. [ $\left.\mathrm{t}^{\mathrm{h}}\right]$, y consonantes totalmente aspiradas, donde la consonante en posición de coda silábica se realiza como una [h].

Las consonantes con fonación aspirada son producidas por un desfasaje en la relajación de los gestos oral y glotal. Luego de la soltura de la oclusión, la glotis permanece abierta antes de la fonación de la vocal permitiendo el paso del aire y provocando la aspiración (Browman y Goldstein 1992). Lo mismo puede ocurrir cuando, como resultado de un proceso morfológico de concatenación de morfemas, se crea una secuencia $\mathrm{VC}_{\text {[oclusiva] }} \mathrm{hV}$, en el cual el gesto glotal abierto de la consonante oclusiva y de la fricativa glotal se superponen de manera parcial en el tiempo y el resultado es el desfasaje entre la soltura del gesto oral y el del gesto glotal.

(9) $\mathrm{C} \rightarrow \mathrm{C}^{\mathrm{h}} / \mathrm{V}-\mathrm{hV}$

Los ejemplos presentados en (10 a-f) ilustran el proceso representado en (9), en los cuales la aspiración ocurre al combinarse un sufijo o clítico que inicia con $/ \mathrm{h} /$.
a. $\mathrm{C}+\mathrm{CVC}+\mathrm{CVC}$
$[\mathrm{n}-]+[\mathrm{paq}]+[=$ hen $]$
$\begin{array}{ll}\rightarrow & \text { C.CV.CVC } \\ \rightarrow & \text { [n.pa.qhen }]\end{array}$ $1 \mathrm{SUJ}+$ teñir$+\mathrm{PL}$
b. $\mathrm{CV}+\mathrm{C}+\mathrm{CV}+\mathrm{CCVC}+\mathrm{CV}$
$\rightarrow$
$[$ ha- $]+[$ n- $]+[$ ti- $]+[$ sqat $]+[-h i] \rightarrow$
CV.Ç.CVC.CV.CV
NEG+1SUJ+RFL+ocultar+NEG
'Yo no me escondo'
c. C.CVC.CV
$\begin{array}{ll}\rightarrow & \text { C.CV.CV } \\ \rightarrow & {\left[\text { n.lo.t }{ }^{\mathrm{h}} \mathrm{u}\right]}\end{array}$
'Lo escucho por dentro'
[n- $]+[$ lot $]+[-\mathrm{hu}]$
1SUJ+sentir+LOC 

d. $\mathrm{C}_{1}+\mathrm{CVC}+\mathrm{CVC}$
$\rightarrow$
C.CV.CVC
$[\mathrm{n}-]+[\mathrm{jik}]+[=\mathrm{hen}]$
$\rightarrow \quad\left[\right.$ n.j.j...$^{h}$ en $]$
'Nosotros nos vamos'
$1 \mathrm{SUJ}+$ irse $+\mathrm{PL}$
e. $\mathrm{CV}+\mathrm{C}+\mathrm{CVC}+\mathrm{CV}$
$\rightarrow \quad \mathrm{CV}+\mathrm{C}+\mathrm{CV}+\mathrm{CV}$
[ha- $]+\left[n_{-1}\right]+\left[\right.$ t'ek$\left.^{\prime}\right]+[-\mathrm{hi}]$
$\rightarrow$
[ha.n..t'e.čhi]
'Yo no como'
$\mathrm{NEG}+1 \mathrm{SUJ}+$ comer+NEG
f. $\mathrm{CVC}+\mathrm{CV} . \mathrm{CV}$
[jik]+[-hi.la]
3SUJ:irse+FUT
$\rightarrow \quad$ CV.CV.CV
$\rightarrow \quad$ [ji.č $\left.{ }^{\mathrm{h}} \mathrm{i} . \mathrm{la}\right]$
'Él/ella se va a ir'

Nótese que en (10d) - (10f) ocurre además un proceso de palatalización, como se explicó en §3.1. En la resilabación el segmento aspirado ocupa la posición de ataque de la sílaba.

La aspiración evita, por un lado, la combinación de dos consonantes contiguas en límite silábico, CVC.CV (al igual que el proceso de ocultamiento de la glotal fricativa) ${ }^{2}$ y, por el otro, una secuencia de sonidos que viola el principio universal de estructura de sílaba (en el cual el ataque es más sobresaliente que la coda) puesto que el sonido en coda es oclusivo y el sonido en ataque es fricativo, más bajo en la escala de sonoridad entre las obstruyentes.

El otro proceso de aspiración que ocurre en wichí afecta a las consonantes nasales en posición de coda silábica seguidas de la semiconsonante palatal en límite morfológico. Este proceso ocurre especialmente cuando se añade el sufijo causativo que inicia con la semiconsonante /j/: /-jen/.
a. $\mathrm{CV}+\mathrm{CV} . \mathrm{CVC} .+\mathrm{CVC}$
CV.CV.CVh ${ }^{\mathrm{h}}$ CVC
[?i] + [qa.tin $]+[-$ jen $]$

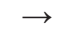
[?i.qa.tin ${ }^{\text {h.jen] }}$
'Lo hace saltar'
b. $\mathrm{CV}+\mathrm{CV} \cdot \mathrm{CVC}+\mathrm{CVC}$
CV.CVh.$C V C$
$[$ ?i- $]+[$ wen $]+[-$ jen $]$
$\rightarrow \quad$ [?i.we $\left.{ }^{\mathrm{h}} . j \mathrm{jen}\right]$
'Hace que lo encienda'
CVBL+encender+CAUS
c. $\mathrm{CV}+\mathrm{CV} \cdot \mathrm{CVC}+\mathrm{CVC}$
CV.CV.CVh.$C V C$
[?i-]+['wa.tshan]+[-jen]
[?i.'wa.tsh $\left.a^{h} . j e n\right]$
'Lo hace verde'
CVBL+ser_verde+CAUS

Asimismo, la aspiración puede producirse después de la vocal y antes de la semiconsonante del mismo sufijo causativo, como se ilustra en (12). Este proceso, que ocurre en el mismo límite morfológico que en los ejemplos de (11 a-e), contribuye al realce de la semiconsonante palatal en ataque silábico y morfológico.

a. $\mathrm{CV}+\mathrm{CV} \cdot \mathrm{CV}+\mathrm{CVC}$

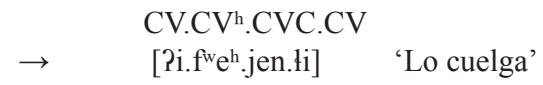

$[? i-]+\left[f^{w} e .1 i\right]+[-j e n]$

\section{CVBL+colgarse+CAUS}

\footnotetext{
${ }^{2} \mathrm{La}$ estructura silábica preferida en el interior de la palabra fonológica es CV.CV.
} 
b. $\mathrm{CV} . \mathrm{CV}+\mathrm{CVC}$

$[$ hi.č́a] $+[-j e n]$

3SUJ:inundarse+CAUS

c. $\mathrm{CV}+\mathrm{CVC}+\mathrm{CV}$

$\left[\mathrm{t}^{\prime} \mathrm{e}\right]+[-\mathrm{jen}]+\left[-\mathrm{k}^{\mathrm{w}} \mathrm{e}\right]^{3}$
CV.CVh.CVC

[hi.č'ah.jen] 'Lo inunda'

$\mathrm{CV}^{\mathrm{h}} \cdot \mathrm{CVC} \cdot \mathrm{CV}$

$\left[\mathrm{t}^{\mathrm{\prime}} \mathrm{e}^{\mathrm{h}} \cdot \mathrm{jen} \cdot \mathrm{k}^{\mathrm{w}} \mathrm{e}\right]$
'Le hace buscar algo'

\subsection{Ensordecimiento de sonorantes}

La serie de nasales y semiconsonantes sordas $/ \mathrm{n} /, / \mathrm{j} / \mathrm{y} / \mathrm{w} /$ forman parte del inventario fonológico del wichí (cf. §2). Pero además, al igual que las oclusivas aspiradas, pueden ocurrir como resultado de un proceso de ensordecimiento por la presencia de la glotal fricativa (con excepción de la semiconsonante velar porque no ocurre en posición de coda). El mismo proceso de ensordecimiento también ocurre con la nasal bilabial, a pesar de que la realización sorda de ésta no haya sido considerada con estatus fonémico.

(13) $\mathrm{C}[$ sonorante $] \rightarrow \mathrm{C} / \mathrm{V}$ $\mathrm{hV}$

Cualquiera de las sonorantes $/ \mathrm{m} /, / \mathrm{n} /, / \mathrm{j} /$ en posición de coda seguida por la glotal fricativa que ocupa el ataque de la sílaba siguiente se ensordece. La sonorante ensordecida pasa a ocupar la posición de ataque; véanse ejemplos en (14).
a. CV.CVC+CVC
$\rightarrow \quad$ CV.CV.CVC
[?i.loj]+[-hen]
$\rightarrow \quad$ [2i.lo.jen]
'Ellos viven'
3SUJ:vivir+PL
b. $\mathrm{C}+\mathrm{CVC}+\mathrm{CVC}$
$\begin{array}{ll}\rightarrow & \text { C.CV.CVC } \\ \rightarrow & \text { [n.či.men }]\end{array}$
'Tenemos sed'
[n- $]+[$ čim $]+[=$ hen]
1SUJ tener_sed PL
c. C.CVC.CV

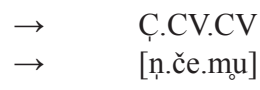
$[n-]+[$ čem $]+[-h u]$
‘Él/ella lo junta (líquido)’
d. $\mathrm{CV}+\mathrm{C}+\mathrm{CVC}+\mathrm{CV}$
$\rightarrow \quad$ CV.C..CV.CV
[ha-]+[n-]+['wen]+[-hi]
$\rightarrow$
[hã.ṇ.'we.noi]
'No lo veo'

\footnotetext{
${ }^{3}$ El ejemplo (12 c) es una raíz verbal colexicalizada con el sufijo direccional $/-\mathrm{k}^{\mathrm{w}} \mathrm{e} / \mathrm{que}$, a pesar de ello, conserva su posición en la estructura morfológica. Por tal motivo, cuando otro sufijo se añade a la base verbal, lo hace entre la raíz y el sufijo colexicalizado. Dado que sincrónicamente es muy difícil reponer el significado básico de la raíz y que es inseparable, ha sido glosado como una raíz discontinua repitiendo su significado en cada una de las partes discontinuadas.
} 
Al igual que la palatalización, la dirección del proceso de ensordecimiento es de derecha a izquierda, pero a diferencia de aquel, este proceso atraviesa el límite silábico. El ensordecimiento ocurre en función de la estructura de sílaba permitida en wichí y del principio de estructura silábica. La nasal en posición de coda está más alta en la escala de sonoridad que la glotal fricativa que se encuentra en posición de ataque. Según el principio de estructura silábica, el ataque debe ser más sobresaliente que la coda. La consonante ensordecida en posición de ataque además forma una secuencia CV.CV preferida en interior de palabra.

\subsection{Ocultamiento de la glotal fricativa}

Las fricativas uvular y glotal en interior de palabra no ocurren en posición de ataque precedidas de una consonante, la ocurrencia de estos sonidos es en posición intervocálica. Esta restricción fonotáctica motiva determinados cambios fonológicos. Uno de ellos es el ocultamiento de la glotal fricativa / $\mathrm{h}$ / cuando está precedida por otra consonante fricativa: $\left[\mathrm{f}^{\mathrm{w}}, \mathrm{s}, 1, \chi\right]$, véase regla en (15).

$$
/ \mathrm{h} / \rightarrow \varnothing / \mathrm{C}[\text {-sonora, }+ \text { cont }]
$$

La apertura de la glotis en la producción de la consonante fricativa sorda en coda se superpone con el mismo gesto articulatorio de la /h/ en ataque silábico, y en consecuencia, la glotal fricativa no se produce en una medida de tiempo posterior a la consonante oral. Los ejemplos presentados en (16 a-d) ilustran este proceso.

\begin{tabular}{|c|c|c|c|}
\hline 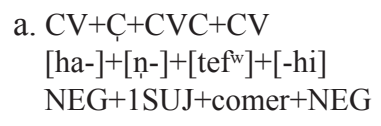 & $\rightarrow$ & $\begin{array}{l}\text { CV.Ç.CV.CV } \\
\text { [hã.n..te.fwi] }\end{array}$ & 'No lo como' \\
\hline $\begin{array}{l}\text { b. } \mathrm{C}_{1}+\mathrm{CVC}+\mathrm{CVC} \\
{\left[\mathrm{n}_{1}-\right]+\left[\mathrm{k}^{\mathrm{w}} \mathrm{es}\right]+[=\text { hen }]} \\
\text { 1SUJ+cortarse }+\mathrm{PL}\end{array}$ & $\rightarrow$ & $\begin{array}{l}\text { C..CV.CVC } \\
{\left[n \cdot k^{\text {we }} \cdot \operatorname{sen}\right]}\end{array}$ & 'Nos cortamos' \\
\hline $\begin{array}{l}\text { c. } \mathrm{CVC}+\mathrm{CV} \\
\text { [tol]+[-hu] } \\
\text { 3SUJ:surgir+LOC }\end{array}$ & $\rightarrow$ & $\begin{array}{l}\mathrm{CV}-\mathrm{CV} \\
\text { [to.tu] }\end{array}$ & 'proviene de' \\
\hline $\begin{array}{l}\text { d. CV.CVC+CV } \\
\quad\left[\text { juk }^{\mathrm{w}} \mathrm{a} \chi\right]+[=\mathrm{hi}] \\
\text { 3SUJ:morder+LOC.en }\end{array}$ & $\rightarrow$ & $\begin{array}{l}\text { CV.CV.CV } \\
{\left[j u . k^{w} a \cdot \chi I\right]}\end{array}$ & 'Mastica algo' \\
\hline
\end{tabular}

En los ejemplos de (16) el símbolo ‘+' indica el límite morfológico y los puntos separan las sílabas. En (16 a) se ilustra el ocultamiento de la glotal fricativa ante la fricativa labial / $\mathrm{f}$ /, en (16 b) y (16 c) el mismo proceso ante la fricativa alveolar $/ \mathrm{s} /$, en $(16 \mathrm{~d})$, ante la fricativa lateral /1/ y en (e)-(g), ante la fricativa uvular $/ \chi /$. Nótese que el mismo proceso ocurre sin importar el morfema que haya sido añadido: negación, plural verbal, causativo, locativo, futuro. Ello indica que el proceso no está circunscripto a un 
morfema particular sino motivado por restricciones fonotácticas en la formación de una palabra fonológica.

\subsection{Fortalecimiento de consonantes}

El fortalecimiento fonológico de un segmento (fortition) es el proceso contrario del debilitamiento (lenition); cf. Brandão de Carvalho, Scheer y Ségéral (2008). El fortalecimiento/debilitamiento supone que las consonantes pueden ser comparadas en términos relativos según sean más/menos fuertes (Honeybone 2008) y puede estar motivado por factores fonológicos o morfosintácticos (Scheer y Ségéral 2008; Bye y de Lacy 2008; Shiraishi 2008; entre otros). Cuando la alternancia del segmento ocurre en límites morfológicos internos de la palabra fonológica, tal como se observa en wichí, las consonantes en posición intervocálica y de postcoda pueden fortalecerse, p.ej. (17-20):

$\begin{array}{llll}\text { CV.CVC+CV } & & \text { CV.CV.CV } & \\ {[\text { la.tes }]+[- \text {-et }]} & \rightarrow & {[\text { [la.te.tsel }]} & \text { 'troncos' *la.te.set }\end{array}$

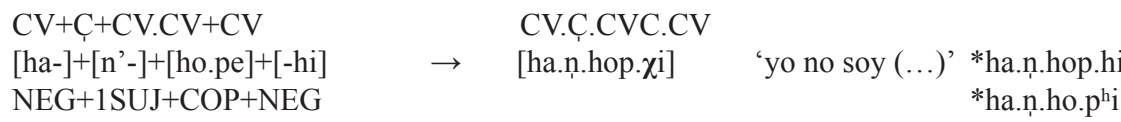

(19)
$\mathrm{CVC}+\mathrm{CVC}$
CVC.CVC
['jet]+[-hat]
$\rightarrow \quad$ ['jet.kat]
'lo cansó' $\quad \begin{aligned} & * \text { 'jet.hat } \\ & * \text { 'je.lat }\end{aligned}$
3SUJ:estar_cansado+CAUS
$\mathrm{C}+\mathrm{CVC}+\mathrm{CV} . \mathrm{CV}+\mathrm{CVC}$
$[\mathrm{n}]+[\mathrm{t}$ 'a $]+[$ hĩ.la $]+[$ ?am $]$
1SUJ+pegar+ FUT $+2 \mathrm{OBJ}$
C.CVC.CV.CV.

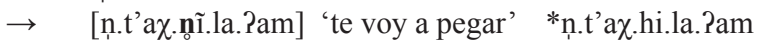
*n.t'a.xi.la.Pam

(20)

Según el gráfico 1 propuesto por Lass (1984: 178) la dirección del cambio de la consonante en el ejemplo (17) es de una oral fricativa (3a) a una africada (4a) en contexto intervocálico, cuya vocal precedente es anterior V $[\mathrm{e}, \mathrm{i}]$ _V (contrástese con /čo.set/ chos-elh [cola-PL] 'colas'). La fricativa fortalecida a africada pasa a ocupar la posición de ataque de la sílaba. En el ejemplo (18) el cambio de la consonante es de una glotal fricativa (2a) a una oral fricativa (3a) en posición de postcoda, cuya consonante en coda silábica es oclusiva C[oclusiva]_V. El proceso esperable en este contexto es el de la aspiración, como se explicó en \$3.3, sin embargo, de producirse la aspiración de la /p/, la cópula /hope/ quedaría prácticamente fusionada con el sufijo negativo. Por último, en los ejemplos (19) y (20) la consonante cambia de (2a) a (5a) según el esquema. En (19) hay, además, una disimilación en cuanto al modo de articulación (fricativa-oclusiva). Nótese que la consonante precedente también es fricativa $C\left[\right.$ fricativa] $V_{\text {, }} \mathrm{y}$ en este contexto lo 
esperable es que ocurra un proceso de ocultamiento de la glotal, como se explicó en $\S 3.5$. En (20) la glotal fricativa, (2a) en el gráfico, en posición de postcoda cambia hacia el otro extremo de la escala, una oclusiva sonora ensordecida, posición (5a) en el gráfico.

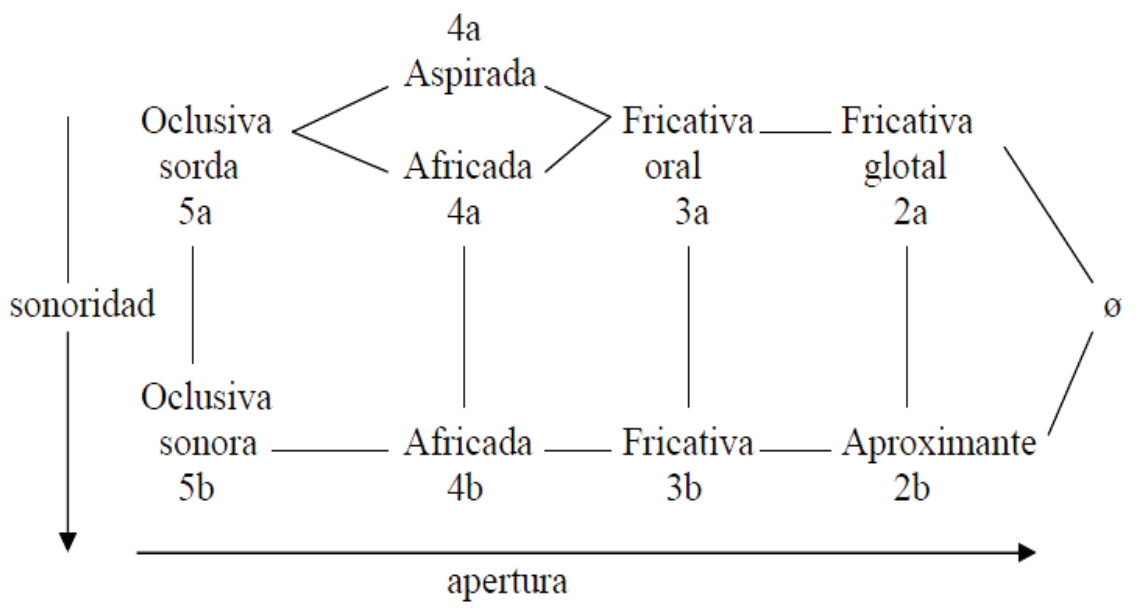

Gráfico 1. Direcciones del fortalecimiento y debilitamiento de consonantes

Hasta el momento no he identificado los factores que motivan el fortalecimiento de manera regular y predecible. Sin embargo, puede observarse que este proceso favorece la transparencia semántica de la palabra en la medida que visibiliza los límites morfológicos internos; mientras que los condicionamientos fonotácticos no parecen tener incidencia.

\subsection{Reducción silábica}

La reducción silábica o síncopa en wichí puede consistir en la supresión de una sílaba entera o en la reducción de una sílaba a un segmento consonántico. A diferencia de los otros procesos morfofonológicos que dependen de restricciones fonotácticas o de estructura de la sílaba, la reducción silábica parece estar motivada por factores rítmico-discursivos. Si bien no he encontrado aún una explicación que consiga predecir y sistematizar este tipo de fenómeno, he hallado evidencias que permiten sostener esta hipótesis. Por un lado, la reducción de la sílaba a un segmento consonántico es sincrónicamente variable, es decir, que puede probarse que en el habla espontánea y rápida los prefijos de persona son producidos en su forma fonológica $/ \mathrm{CV} /$ o reducida $[\mathrm{C}]$ en un mismo texto y por el mismo hablante. Asimismo, los sustantivos en posición de objeto verbal pueden ser producidos en su forma entera o reducida a una sola sílaba que se clitiza a la raíz verbal formando un compuesto $\mathrm{V}+\mathrm{N}$. Por otro lado, la reducción silábica de las palabras derivadas, en las cuales dicho proceso está actualmente cristalizado (es decir, no aceptan la forma no reducida) no se restringe ni a una clase de palabra en particular, ni a un tipo de derivación o proceso de formación de palabra específico, ni a un tipo de afijo determinado. Por último, las estructuras fonológicas sin la reducción silábica son estructuras posibles, existentes y aceptables, es decir que no violan ningún tipo de restricción o condicionamiento fonotáctico o de la estructura silábica o de palabra que motive la síncopa. Así pues, se 
especula que los procesos de síncopa ya cristalizados, posiblemente por frecuencia de uso, hayan sido originados por los mismos factores rítmico-discursivos que sincrónicamente motivan la reducción silábica en el discurso.

Una de las formas de síncopa que tiene como resultado la reducción de una sílaba completa es mediante la elisión de un segmento vocálico lo que, en la resilabación, da como resultado una palabra con una sílaba menos. La tendencia es suprimir la vocal de la primera sílaba de la raíz contigua al afijo añadido: la primera de la raíz si es un prefijo y la última de la raíz si es sufijo. Nótese que la adición de los prefijos pronominales de persona no provoca este proceso. En (21) se presentan algunos ejemplos de bases verbales y en (22) de bases nominales.

a. $\mathrm{CV}+\mathrm{CV} \cdot \mathrm{CVC}+\mathrm{V}$

$[$ ni- $]+[$ pi. 'ta $]+[-\mathrm{a}]$

$\mathrm{NEG}+$ ser_alto+NEG

b. CV+CV.CV.CV.CV

[to-]+[ni.' to.fwe.lex]

SUJ.INDF+conocer

c. CV.CVC+CV.CV

[jr.' set]+[-hi.la]

3SUJ:cortar+FUT

d. CV.CVC+CVC

[jr. 'set] $+[$-hen]

3SUJ:cortar+PL

e. $\mathrm{C}+\mathrm{CVC}+\mathrm{CV} \cdot \mathrm{CV}+\mathrm{CV}+\mathrm{CVC}$

$[$ n- $]+[$ t'os $]+[-$ hi.la $]+[-$ ?a $]+[$ 'pe? $]$

$1 \mathrm{SUJ}+$ pisar+FUT+2OBJ+pisar

\begin{tabular}{|c|c|c|}
\hline$\rightarrow$ & $\begin{array}{l}\text { CVC.CV.CV } \\
\text { [nip.'ta. } \chi a]\end{array}$ & 'No es alto' \\
\hline$\rightarrow$ & $\begin{array}{l}\text { CVC.CV.CV.CV } \\
\text { [ton.' to.fwe.lex] }\end{array}$ & 'Se lo conoce' \\
\hline$\rightarrow$ & $\begin{array}{l}\text { CVC.CV.CV } \\
\text { [jis. } \text { thi. 'la] }^{\text {hi }}\end{array}$ & 'Lo va a cortar \\
\hline$\rightarrow$ & $\begin{array}{l}\text { CVC.CVC } \\
\text { [jis.' } \text { then] }^{\text {hen }}\end{array}$ & 'Los corta' \\
\hline$\rightarrow$ & $\begin{array}{l}\text { C..CV.CV.CV.CVC } \\
\text { [n., tsi.la., ?a.'pe?] }\end{array}$ & 'Te voy a pisar' \\
\hline
\end{tabular}

CV.CVC

$\rightarrow \quad$ [č'a.'plis] 'platos'

[č'a.'pel] $+[$-is $]$

plato $+\mathrm{PL}$

b. CV.CVC+VC

[hu.'san] $+[-$ is $]$

hacha + PL

c. CV.CVC+VC

[qu.set] $+[$-es]

pantalón $+\mathrm{PL}$

d. $\mathrm{CV}+\mathrm{CV} . \mathrm{CVC}+\mathrm{VC}$

$[$ to- $]+[$ te.nek $]+[-$ aj $]$

POS.INDF+canción+PL
CVC.CVC

$\rightarrow \quad$ [hus.'nis] 'hachas'

$\rightarrow \quad$ Cqus.'tes] 'pantalones'

CV.CVC.CVC

$\rightarrow \quad$ [to.ten.kaj] 'canciones' 


$\begin{array}{llll}\begin{array}{l}\text { e. } \mathrm{CV}+\mathrm{CV} . \mathrm{CVC}+\mathrm{VC} \\ \text { [to-]+[po.'tsin]+[-ek] }\end{array} & \rightarrow & \begin{array}{l}\text { CV.CVC.CVC } \\ \text { [to.pot.'nek] }\end{array} & \text { 'trenza' } \\ \text { POS.INDF+armar+NMLZ } & & \\ & & \\ \text { f. CV+CV.CV.CVC+VC } & & \\ & \text { CV.CVC.CVC } & \\ \text { [to-]+[ji.he.min]+[-ek] } & \rightarrow & \text { [to.hem.'nek] } & \text { 'amante' } \\ \text { POS.INDF+gustar/amar+NMLZ } & & & \end{array}$

La cantidad de sílabas de la palabra fonológica resultante de este proceso de síncopa es muy variable, puede contener de dos a cuatro sílabas. No obstante, en todos los casos la reducción es de una sílaba con respecto a la palabra derivada sin la reducción. Nótese que la vocal elidida puede ser la acentuada o no, por lo que el acento tampoco tiene injerencia en este proceso. En todos los casos presentados en (21) y en (22) la elisión de la vocal es requerida para la buena formación de la palabra. Es decir, no existe la opción de la palabra sin reducción silábica. Si se sostiene la hipótesis de que el proceso de síncopa es debido a factores rítmico-discursivos, entonces, estos son casos en los que la reducción silábica se ha cristalizado.

Otra forma de síncopa consiste en la supresión de la sílaba completa, generalmente átona, en la formación de compuestos. Este mecanismo es altamente frecuente en los textos y es muy productivo para la formación de palabras nuevas, por tal motivo se encuentran compuestos nominales con este tipo de estructura y, al igual que en la reducción vocálica, está cristalizado como parte del proceso morfofonológico de formación de la palabra. Los ejemplos presentados en (23) son compuestos nominales que presentan la reducción silábica como requisito para la buena formación de la palabra. a. [?i.'pe?]+[?i.'not]
3SUJ:estar_encima+agua
$\rightarrow \quad$ [2i.pe?.'not] $\quad$ 'flotar'
b. ['ma.jek]+[wi.'t'oj] $\rightarrow \quad$ [maq., wi.'t'oj] 'enfermedad' algo+doler
c. [ha.' 'lo]+[maq.' wu] árbol+insecto
$\rightarrow \quad[$ ha., 'lo.'wu $] \quad$ 'termita'
d. [to-]+[č'u'te?]+[ti'san] POS.INDF+oreja+carne
$\rightarrow \quad[$ to. . ču.te.'san]
'lóbulo de la oreja'
e. [to-]+['wu]+[la.'tes] POS.INDF+cuello+inicio
$\rightarrow \quad$ [to.,'wu.'tes] 'nuca'

En (24) se presentan ejemplos extraídos de textos narrativos que ilustran el mismo tipo de síncopa y composición. A diferencia de los ejemplos presentados en (23), la reducción silábica en (24) es estilística o rítmica a nivel discursivo, por lo tanto la forma completa y la reducida pueden alternar. 
(24)
a. ['t'e.k ${ }^{w} e$ \# ?i. 'not]
[,'e.kwe.'not]
'Busca agua'
buscar\#agua
b. [hi.,p'ox.' li \# hu. 'nat]
pegar piñas\#suelo
[hi.,p'ox.1i. 'nat]
'Pegaba piñas al piso'
c. [ta. 'če.mu \# Pi. 'not]
[ta., če.mou.'not]
'Juntaba agua'
agarrar\#agua

La reducción de la sílaba de una palabra y la combinación de esa con otra forma una palabra fonológica recursiva compleja ${ }^{4}$. El acento y la reducción misma contribuyen a la integración de los dos miembros del compuesto, además de la proximidad sintácticosemántica, la mayoría de las veces se trata de un verbo y su objeto. La frecuencia de uso de este tipo de construcciones puede dar como resultado la integración de ese compuesto al vocabulario de la lengua como unidad colexicalizada o cristalizada.

La frecuencia de uso de este tipo de síncopa como recurso prosódico-discursivo es una fuente de origen para las innovaciones léxicas y aproxima la distancia entre la frase y la palabra como unidades textuales.

Un tercer tipo de síncopa no reduce la cantidad de sílabas de la palabra pero sí suprime la vocal de la sílaba y mantiene la consonante como núcleo silábico. Este tipo de proceso ocurre especialmente con los prefijos de persona, que son monosilábicos. El segmento que se suprime es el núcleo vocálico del prefijo quedando como elemento morfológico la consonante.

En el habla rápida algunos prefijos, como los de persona sujeto y poseedor, pueden ser producidos en su forma completa $/ \mathrm{CV} /$ o reducida $[\mathrm{C}]$. Las dos formas pueden aparecer en un mismo texto y producido por el mismo hablante.
a. [la- $]+[$ 'wu $]$ 3POS+cuello
$\rightarrow \quad[1 \mathrm{la} \cdot \mathrm{wu}] \sim\left[1,{ }^{\prime} \mathrm{wu}\right]$
b. [la- $]+[$ pa.č'u]
$\rightarrow \quad[$ la.pa.č'u $] \sim[1$. pa.č'u $]$
3POS+pie
c. $[$ la- $]+[$ les $]$
3POS+hijo
$\rightarrow \quad[$ la.les $] \sim[1$. les $]$

Los ejemplos presentados en (25) corresponden todos al prefijo de tercera persona poseedor porque es el único prefijo $\mathrm{CV}$ cuya consonante en ataque es sonorante y puede ocupar la posición de núcleo silábico. Pero el mismo proceso también puede observarse en otros prefijos que actualmente han quedado reducidos y perdieron su forma alternativa completa.

\footnotetext{
${ }^{4}$ Una palabra fonológica que contiene otra palabra fonológica.
} 
Uno de estos casos es el prefijo de primera persona sujeto. A mediados del siglo XIX, Remedi (1896: 356) y Pelleschi (1897: 250) registraron las formas /'no-/ /'nu-/ para la primera persona sujeto y poseedor. En el siglo XX, casi cien años más tarde, la variedad bermejeña presenta la forma reducida /'n:-/ (Golluscio 1990, m.i.), y la pilcomayeña /õ:-/ (Tovar 1961). La diferencia entre las dos formas reducidas, /'n:-/ y /õ:-/, de la variedad bermejeña y pilcomayeña respectivamente se constatan con los datos registrados en la actualidad.

Otro caso de reducción diacrónica es el del prefijo de clase verbal intransitiva /ta-/. Aunque este proceso parece ser más antiguo aún, la evidencia de la reducción sale a la luz en la flexión de los verbos según cambien los prefijos de persona sujeto. Cuando las raíces verbales inician con consonantes, el prefijo /ta-/ sólo es reducido a/t-/ en la primera persona puesto que el prefijo nasal silábico ocupa la posición de núcleo y la oclusiva alveolar puede formar sílaba con esa en posición de coda. Mientras que en la segunda persona y en la tercera se mantuvo en su forma completa, véanse ejemplos en (26) y (27).

\begin{tabular}{|c|c|c|}
\hline a. [nt.qa.tin] & $<$ & $\begin{array}{l}\text { nu-ta-qa.tin } \\
\text { 1SUJ-CVBL-saltar }\end{array}$ \\
\hline b. [la.ta.qa.tin] & $<$ & $\begin{array}{l}\text { la-ta-qa.tin } \\
\text { 2SUJ-CVBL-saltar }\end{array}$ \\
\hline c. [ta.qa.tin] & $<$ & $\begin{array}{l}\varnothing \text {-ta-qatin } \\
\text { 3SUJ-CVBL-saltar }\end{array}$ \\
\hline d. [tot.qa.tin] & $<$ & $\begin{array}{l}\text { to-ta-qatin } \\
\text { 1SUJ.PL-CVBL-saltar }\end{array}$ \\
\hline a. [nt.qo.lo] & $<$ & $\begin{array}{l}\text { n-ta-qolo } \\
\text { 1POS-CNML-pierna }\end{array}$ \\
\hline b. [?at.qo.lo] & $<$ & $\begin{array}{l}\text { Pa-ta-qolo } \\
\text { 2POS-CNML-pierna }\end{array}$ \\
\hline c. [ta.qo.lo] & $<$ & $\begin{array}{l}ø \text {-ta-qolo } \\
\text { 3POS-CNML-pierna }\end{array}$ \\
\hline
\end{tabular}

En raíces verbales que inician con PV, el morfema /ta-/ reducido a /t-/ se fusionó con la primera sílaba de la raíz en la posición de ataque formando una sola sílaba. La manera de reconocer este límite morfológico puede ser mediante la elicitación de la forma de imperativo (que consiste en la raíz verbal despojada de prefijos, con excepción del hortativo) y la derivación causativa, que requiere el cambio del clasificador verbal de clase monovalente a bivalente (Vidal y Nercesian 2005). Los ejemplos presentados en (28) ilustran el contraste de la forma en tercera persona con la imperativa y los presentados en (29) con la derivación causativa. 
(28) Indicativo
a. $[\varnothing-\mathrm{ta}-\mathrm{Pek}]>$ [t'ek] 'come'
b. [ø-ta-Piwin] $>$ [t'i.win] 'galopa'
c. [ø-ta-Pisčej] $>$ [t'is.čej] 'se ríe'
d. [ø-ta-?on] $>$ [t'on] 'grita'

(29) forma básica
a. [ø-ta-wajej] 'se casa'
[ø-Pi-waje-jen] 'hace que se case'
b. [ø-ta-fwuče] 'se abre'
[ø-Pi-fwu-jen-če] 'hace que se abra'
e. [ø-ta-qataj] 'cocina'
[ø-Pi-qata ${ }^{\mathrm{h}}$-jen] 'hace que cocine'

Es probable que la reducción silábica sea un fenómeno que caracterice a las otras lenguas mataguayas e incluso a otras lenguas del área, dado que la síncopa también se ha registrado en el pilagá de la familia guaycurú (Vidal 2001: 50ss.). Un estudio cuidadoso de los niveles textual y prosódico permitirá explicar de qué manera la reducción silábica está vinculada a patrones rítmico-discursivos como se especula aquí.

\section{LOS PROCESOS FONOLÓGICOS COMO REQUISITO DE FORMACIÓN DE PALABRA Y LA PALABRA COMO DOMINIO DE LOS PROCESOS}

Existe una relación casi "recíproca" entre los procesos fonológicos y la palabra fonológica en tanto los procesos fonológicos analizados ocurren dentro de la palabra fonológica y para su formación y, al mismo tiempo, la palabra fonológica es el dominio en el que ocurren esos procesos. Los ejemplos que se presentan a continuación comparan el ocultamiento de la fricativa glotal y la palatalización dentro de la palabra (30a y 31a) y su bloqueo en presencia de un límite léxico (30b y $31 \mathrm{~b}$ ).

(30)

\section{a. Ocultamiento de la fricativa glotal}

$[\mathrm{n}-]+[\mathrm{paq}]+[-\mathbf{h e n}]$

$\rightarrow \quad\left[\right.$ n.p.p.q. $\left.q^{\text {hen }}\right]$

'Yo los teñí'

$1 \mathrm{SUJ}+$ teñir$+\mathrm{PL}$

\section{b. Bloqueo de la regla entre palabras}

...[ña.na.jex\#tuq\#ho.pe]...

NEG.3SUJ.IRR:saber:NEG:saber\#PRO.INDF\#COP

...'no sabían quién era'... 
(31)

\section{a. Palatalización}

[?i.wu.je]+[te.nek]+[-a] $\rightarrow \quad$ [?i.wu.te.ne.ča] 'adora'

3SUJ:hacer+canción+CI

\section{b. Bloqueo de la regla entre palabras}

i) $\quad \ldots$ [na.qu.jex\#?a.qa.pi.ni] ...

HORT:jugar:INS\#tu:miel

... 'apostemos tu miel'...

ii) $\quad \ldots[$ wit\#net.ta.lek\#?i.not]...

CONJ\#3SUJ:aguantar\#agua

... 'y el que aguanta en el agua'...

Este contraste permite reconocer los límites de la palabra, y en consecuencia, los procesos fonológicos sirven para su definición. Al mismo tiempo, la relación circular entre los procesos y la palabra como dominio es un indicio de que ocurren simultáneamente a la combinación morfológica en la formación de palabras y no en un ordenamiento en niveles (Nercesian 2011c).

\section{CONCLUSIONES}

La palabra fonológica en wichí constituye el dominio en el que ocurren diversos procesos fonológicos que manifiestan frecuencia y regularidad. Se analizaron (i) la palatalización, (ii) la epéntesis vocálica y consonántica, (iii) la aspiración de obstruyentes, (iv) el ensordecimiento de sonorantes, (v) el ocultamiento de la glotal fricativa, (vi) el fortalecimiento de consonantes y (vii) la reducción silábica. El grado de fusión, no obstante, no impide el reconocimiento y la segmentación morfológica de la palabra, y en consecuencia no opaca la transparencia morfotáctica, alineándose así con la tendencia de las lenguas aglutinantes.

Se observó que los procesos fonológicos están motivados por restricciones y condicionamientos de distintos tipos: a) articulatorios, b) fonotácticos de estructura silábica, c) de sandhi interno, d) de transparencia semántica, y e) rítmicos. Según estos factores condicionantes y el nivel lingüístico que hacen prevalecer, los procesos fonológicos se agrupan de la siguiente manera:

a) la palatalización sigue un condicionamiento articulatorio que tiende a hacer prevalecer las restricciones del nivel del segmento;

b) la epéntesis vocálica y consonántica están motivadas por la fonotaxis que tiende a hacer prevalecer las condiciones de estructura de la sílaba; 
c) la aspiración de obstruyentes, el ensordecimiento de sonorantes y el ocultamiento de la glotal fricativa siguen las restricciones del sandhi interno que da prioridad a la combinación de sonidos entre sílabas dentro de la palabra;

d) el fortalecimiento de consonantes ocurre en favor de la transparencia semántica que da prioridad a la estructura de la palabra gramatical; y

e) la reducción silábica motivada por factores rítmicos a nivel de la frase.

La palabra es, como se dijo, el dominio en el que ocurren los procesos fonológicos que involucran a todos los niveles lingüísticos y por ello es una unidad compleja y multidimensional, pero al mismo tiempo, esos procesos fonológicos contribuyen a la formación de la palabra. Contribuyen a su integridad como palabra a la vez o en tanto que contribuyen a su formación (si esos procesos fonológicos no ocurren, no resulta una unidad aceptable para los hablantes). Asimismo, se mostró que los procesos que ocurren en interior de palabra no ocurren entre palabras dentro de una frase, y en tal sentido permiten delimitar la unidad como dominio lingüístico morfofonológico (fonológico y gramatical).

Finalmente, a partir de este estudio es posible profundizar otros aspectos que no han sido explorados exhaustivamente en este trabajo como los factores rítmicos que motivan la reducción silábica y la comparación de estos procesos fonológicos con los de la variedad pilcomayeña.

\section{REFERENCIAS BIBLIOGRÁFICAS}

BOOIJ, Geert; RUBACH, Jerzy (1987). Postcyclic versus postlexical rules in Lexical Phonology. Linguistic Inquiry 18: 1-44.

BOOIJ, Geert (1999). The role of the prosodic word in phonotactic generalizations. En Alan T. Hall; Ursula Kleinhenz (eds.). Studies on the Phonological Word, pp. 47-72. Amsterdam/Philadelphia: John Benjamins.

BOOIJ, Geert; LIEBER, Rochelle (1993). On the Simultaneity of Morphological and Prosodic Structure. En Sharon Hargus; Ellen Kaisse (eds.). Studies in Lexical Phonology, pp. 23-44. San Diego: Academic Press.

BRANDÃO de CARVALHO, Joaquim; SCHEER, Tobias; SÉGERAL, Philippe (eds.) (2008). Lenition and Fortition. Berlin/New York: Mouton de Gruyter.

BROWMAN, Catherine; GOLSDTEIN, Louis (1986). Towards an articulatory phonology. Phonology Yearbook 3: 219-252.

BROWMAN, Catherine; GOLSDTEIN, Louis (1989). Articulatory Gestures as Phonological Units. Phonology 6: 201-251.

BROWMAN, Catherine; GOLSDTEIN, Louis (1992). Articulatory Phonology: an overview. Phonetica 49: 155-180.

BYE, Patrik; de LACY, Paul (2008). Metrical influences on Fortition and Lenition. En Joaquim Brandão de Carvalho; Tobias Scheer; Philippe Ségeral (eds.) (2008), pp. 173-206.

CLEASSON, Kenneth (1994). A phonological outline of mataco-noctenes. International Journal of American Linguistics 60(1): 1-37. 
NERCESIAN - PROCESOS FONOLÓGICOS EN EL DOMINIO DE LA PALABRA WICHÍ...

DIXON, R.M.W.; AIKHENVALD, Alexandra (2002). Word: a typological framework. En R.M.W. Dixon; Alexandra Aikhenvald (eds.) Word. A cross-linguistic typology, pp. 1-41. Cambridge: Cambridge University Press.

GERZENSTEIN, Ana (1978). Lengua Chorote. Buenos Aires: Universidad de Buenos Aires.

GERZENSTEIN, Ana (1994). Lengua Maká. Estudio descriptivo. Buenos Aires: Universidad de Buenos Aires.

GOLlUSCIO, Lucía (1990). Descripción del núcleo gramatical de la lengua wichí (mataco). Variedad hablada en el Teuco (El Sauzalito, Chaco y zona de influencia). Informe de Avance. Consejo Nacional de Investigaciones Científicas y Técnicas (m.i.).

GONZÁLEZ, Hebe (2005). A Grammar of Tapiete (Tupi-Guarani). Tesis de Doctorado. Universidad de Pittsburgh.

GUALDIERI, Beatriz (1998). Mocoví (Guaicuru). Fonologia e morfossintaxe. Tesis de Doctorado. Campinas: Universidad de Campinas.

HALL, Alan T. (1999). The Phonological Word: A Review. En Alan T. Hall; Ursula Kleinhenz (eds.) Studies on the Phonological Word, pp. 1-22. Amsterdam/Philadelphia: John Benjamins.

HONEYBONE, Patrick (2008). Lenition, wakening and consonantal strength: tracing concepts through the history of phonology. En Brandão de Carvalho; Tobias Scheer; Philippe Ségeral (eds.) (2008), pp. 9-92.

LASS, Roger (1984). Phonology. An Introduction to Basic Concepts. Cambridge: Cambridge University Press.

MADDIESON, Ian (2011a). Consonant Inventories. En Matthew S. Dryer; Martin Haspelmath (eds.). The World Atlas of Language Structures Online. Munich: Max Planck Digital Library, chapter 1. Available online at http://wals.info/chapter/1 (Accessed on 2013-02-20).

MADDIESON, Ian (2011b). Vowel Quality Inventories. En Matthew S. Dryer; Martin Haspelmath (eds.). The World Atlas of Language Structures Online. Munich: Max Planck Digital Library, feature 2A. Available online at http://wals.info/feature/2A. (Accessed on 2013-02-21).

MADDIESON, Ian (2011c). Syllable Structure. En Matthew S. Dryer; Martin Haspelmath (eds.). The World Atlas of Language Structures Online. Munich: Max Planck Digital Library, feature 12A. Available online at http://wals.info/feature/12A. (Accessed on 2013-02-21).

MESSINEO, Cristina (2003). Lengua toba. Aspectos gramaticales y discursivos. Lincolm Europa: Münich.

NAJLIS, Elena (1966). Lengua abipona. Volúmenes I y II. Buenos Aires: Centro de Estudios Lingüísticos.

NERCESIAN, Verónica (2011a). Gramática del wichí, una lengua chaqueña. Interacciones fonologíamorfología-sintaxis en el léxico. Tesis de Doctorado. Universidad de Buenos Aires. (Inédita).

NERCESIAN, Verónica (2011b). Stress in Wichí (Mataguayan) and its Interaction with the Word Formation Processes. Amerindia, $\mathrm{N}^{\circ}$ 35: 75-102.

NERCESIAN, Verónica (2011c). Interacciones morfofonología, morfosintaxis y morfosemántica en el dominio de la palabra en wichí (mataguaya). $R A S A L N^{\circ} 1 / 2$.

NESPOR, Marina; VOGEL, Irene (1986). Prosodic Phonology. Dordecht: Foris.

PELLESCHI, Giovanni (1897). Los índios mataguayos y su lengua (con nota de Lafone Quevedo). Boletín del Instituto Geográfico Argentino. Tomo XVIII. Cuadrenos 4, 5 y 6, pp. 173-350. La Plata: Biblioteca Lingüística del Museo de La Plata. 
REMEDI, Joaquín (1896). Los indios matacos y su lengua. Con vocabulario ordenado por Lafone Quevedo. Boletín Geográfico Argentino. Tirada Aparte. Buenos Aires.

SALTZMAN, Elliot (1995). Intergestural timing in speech production: Data and modeling. Proceedings of the XIIIth International Congress of Phonetic Sciences 2: 84-91.

SALTZMAN, Elliot; KELSO, J. A. Scott (1987). Skilled actions: a task-dynamic approach. Psychologicl Review 94, 1: 84-106.

SANDALO, Filomena (1995). A Grammar of Kadiwéu. Tesis de Doctorado. Universidad de Pittsburgh.

SCHEER, Tobias; SÉGERAL, Philippe (2008). Positional factors in Lenition and Fortition. En Joaquim Brandão de Carvalho; Tobias Scheer; Philippe Ségeral (eds.) (2008), pp. 131-172.

SHIRAISHI, Hidetoshi (2008). Nivkh. En Joaquim Brandão de Carvalho; Tobias Scheer; Philippe Ségeral (eds.) (2008), pp. 387-414.

STELL, Nélida (1987). Gramática descriptiva de la lengua nivacle (chulupí). Tesis de Doctorado. Universidad de Buenos Aires.

TOVAR, Antonio (1961). Catálogo de Lenguas de América del Sur. Buenos Aires: Sudamericana.

VIDAL, Alejandra (2001). Pilagá Grammar (Guaykuruan Family, Argentina). Tesis de Doctorado. Universidad de Oregon.

VIDAL, Alejandra; NERCESIAN, Verónica (2005). Sustantivo y verbo en wichí: Hacia una taxonomía de clases de palabras. Liames 5: 7-25.

\section{ABREVIATURAS}

CAUS=causativo; $\quad \mathrm{CI}=$ cierre $\quad$ de incorporación; $\quad \mathrm{CL}=$ clasificador de posesión; $\mathrm{CNML}=$ clase $\quad$ nominal; $\quad \mathrm{CONJ}=$ conjunción; $\quad \mathrm{COP}=$ cópula; $\quad \mathrm{CVBL}=$ clase verbal; $\mathrm{DEM}=$ demostrativo; ep=epentético; $\mathrm{FUT}=$ futuro; HORT=hortativo; $\mathrm{INCL}=$ inclusivo; INDF=indefinido; INS=instrumento; IRR=irrealis; LOC=locativo; NEG=negación; NMLZ=nominalizador; $\mathrm{OBJ}=$ objeto; $\mathrm{PL}=$ plural; $\mathrm{POS}=$ poseedor; $\mathrm{PRO}=$ pronombre; $\mathrm{RFL}=$ reflexivo; $\mathrm{SUJ}=$ sujeto.

Recebido: 21/3/2013

Versão revista: 1/6/2013

Aceito: 20/6/2013. 